UCI-TR-2004-30

hep-ph/0409278

\title{
Slepton Trapping at the Large Hadron and International Linear Colliders
}

\author{
Jonathan L. Feng and Bryan T. Smith \\ Department of Physics and Astronomy, \\ University of California, Irvine, CA 92697, USA
}

\begin{abstract}
We consider supergravity with a gravitino lightest supersymmetric particle. The next-to-lightest supersymmetric particle (NLSP) decays to the gravitino with lifetime naturally in the range $10^{4}-$ $10^{8} \mathrm{~s}$. However, cosmological constraints exclude lifetimes at the upper end of this range and disfavor neutralinos as NLSPs, leaving charged sleptons with lifetimes below a year as the natural NLSP candidates. Decays to gravitinos may therefore be observed by trapping slepton NLSPs in water tanks placed outside Large Hadron Collider (LHC) and International Linear Collider (ILC) detectors and draining these tanks periodically to underground reservoirs where slepton decays may be observed in quiet environments. We consider $0.1,1$, and 10 kton traps and optimize their shape and placement. We find that the LHC may trap tens to thousands of sleptons per year. At the ILC, these results may be improved by an order of magnitude in some cases by tuning the beam energy to produce slow sleptons. Precision studies of slepton decays are therefore possible and will provide direct observations of gravitational effects at colliders; percent level measurements of the gravitino mass and Newton's constant; precise determinations of the gravitino's contribution to dark matter and supersymmetry breaking's contribution to dark energy; quantitative tests of supergravity relations; and laboratory studies of Big Bang nucleosynthesis and cosmic microwave background phenomena.
\end{abstract}

PACS numbers: 04.65.+e, 12.60.Jv, 26.35.+c, 98.80.Es 


\section{INTRODUCTION}

Weak-scale supersymmetry remains a beautiful framework for resolving the problems of electroweak symmetry breaking and dark matter [1], and its discovery is among the most eagerly anticipated events in particle physics. Opportunities for supersymmetry discoveries and studies at colliders depend largely on which superpartner is the lightest supersymmetric particle (LSP). In simple supergravity models, supersymmetry is transmitted to standard model superpartners through gravitational interactions, and supersymmetry is broken at a high scale. The mass of the gravitino $\tilde{G}$ is

$$
m_{\tilde{G}}=\frac{F}{\sqrt{3} M_{*}}
$$

and the masses of standard model superpartners are

$$
\tilde{m} \sim \frac{F}{M_{*}},
$$

where $F \sim\left(10^{11} \mathrm{GeV}\right)^{2}$ is the supersymmetry breaking scale squared, and $M_{*}=$ $\left(8 \pi G_{N}\right)^{-1 / 2} \simeq 2.4 \times 10^{18} \mathrm{GeV}$ is the reduced Planck scale. The precise ordering of masses depends on unknown, presumably $\mathcal{O}(1)$, constants in Eq. (2). Most supergravity studies assume that the LSP is a standard model superpartner, such as a slepton or neutralino.

Recently attention has turned to the other logical possibility, namely, that the gravitino is the LSP [2, 3, 4, 5, 6, 17, 8, 9, 10, 11, 12]. In supergravity where supersymmetry breaking

is mediated by gravity, the gravitino has a mass $m_{\tilde{G}} \sim 100 \mathrm{GeV}$ if the superpartner mass scale is $\tilde{m} \sim 100 \mathrm{GeV}$. The gravitino couplings are also suppressed by $M_{*}$. The gravitino's extremely weak interactions imply that it is irrelevant for most supersymmetric processes. However, if the gravitino is the LSP, the next-to-lightest supersymmetric particle (NLSP) decays to its standard model partner and a gravitino. The NLSP is a weak-scale particle decaying gravitationally and so has a natural lifetime of

$$
\frac{M_{*}^{2}}{\tilde{m}^{3}} \sim 10^{4}-10^{8} \mathrm{~s}
$$

as will be discussed more fully in Sec. II. This lifetime emerges naturally in this simple supersymmetric scenario. At the same time, it is outlandishly long by particle physics standards [13]. It requires a revamping of many aspects of supersymmetric phenomenology and cosmology and opens up novel opportunities, including the one discussed here.

The gravitino LSP scenario is constrained by cosmological and astrophysical data. The gravitino is a stable superweakly massive particle (superWIMP) and forms dark matter. Its production during reheating and by NLSP decays is therefore constrained by measurements of the non-baryonic cold dark matter density. NLSP decays also deposit electromagnetic 14, 15, 16, 17] and hadronic [18, 19] energy into the universe well after Big Bang nucleosynthesis $(\mathrm{BBN})$, and so may ruin the successful predictions of standard BBN. These decays may also distort the cosmic microwave background (CMB) from its observed Planckian spectrum. Last, photons produced in NLSP decays are subject to bounds on the diffuse photon flux.

The impact of these constraints on the gravitino LSP scenario have been considered in detail. In addition to the leading two-body NLSP decays to the gravitino [2, 3, 4, 5], three-body NLSP decays must also be considered when they are the leading contribution to 
hadronic cascades [7, 8]. The result is that the gravitino LSP scenario is not excluded and, in fact, all constraints may be satisfied for natural weak-scale NLSP and gravitino masses.

Not all possibilities are allowed, however, and two results are particularly worth noting. First, neutralino NLSPs are highly disfavored [8, 12]. Neutralinos typically have two-body decays $\chi \rightarrow Z \tilde{G} \rightarrow q \bar{q} \tilde{G}$. The resulting hadronic cascades destroy BBN successes, and exclude this scenario unless such decays are highly suppressed. Kinematic suppression is not viable, however - if $m_{\chi}-m_{\tilde{G}}<m_{Z}$, the decay $\chi \rightarrow \gamma \tilde{G}$ takes place so late that it violates bounds on electromagnetic cascades. Neutralino NLSPs are therefore allowed only when the two-body decays to $Z$ bosons are suppressed dynamically, as when the neutralino is photino-like, a possibility that is not well-motivated by high energy frameworks. Slepton and sneutrino NLSPs also produce hadronic energy when they decay, but this occurs only through three-body decays. These have been analyzed and found to be safe 7, 8]. As a result, the most natural NLSP candidates are sleptons, particularly the right-handed stau.

Second, cosmological constraints exclude the upper range of lifetimes in Eq. (3) [2, 3]. Very late decays occur in a cold universe where decay products are not effectively thermalized and so are especially dangerous. For typical thermal relic NLSP abundances, the CMB and BBN constraints therefore provide an upper bound on NLSP lifetimes, roughly excluding those above a year.

In passing, we note that the scenario outlined above has a number of other motivations. One such motivation is from BBN. Late NLSP decays not only pass all BBN constraints, they may even resolve the leading BBN anomaly by destroying ${ }^{7} \mathrm{Li}$ to bring the predicted abundance in line with the low values favored by observations [2, 3]. To resolve the ${ }^{7} \mathrm{Li}$ anomaly, the preferred NLSP lifetime is $\sim 3 \times 10^{6} \mathrm{~s}[5]$, that is, about a month. A second motivation follows from considerations of leptogenesis [20]. Gravitinos may be produced during reheating. If the gravitino is not the LSP, its late decays are dangerous to BBN, and require reheating temperatures $T_{\mathrm{RH}} \lesssim 10^{5} \mathrm{GeV}$ to $10^{8} \mathrm{GeV}[19]$, in conflict with the requirement $T_{\mathrm{RH}} \gtrsim 3 \times 10^{9} \mathrm{GeV}$ of thermal leptogenesis [21]. In contrast, in the gravitino LSP scenario, the gravitino does not decay, and the reheat temperature is bounded only by the overclosure constraint on the gravitino density. For $m_{\tilde{G}} \sim 100 \mathrm{GeV}$, reheat temperatures as high as $\sim 10^{10} \mathrm{GeV}$ are allowed [22, 23], consistent with thermal leptogenesis [24, 25]. Additional connections between leptogenesis and gravitino LSPs are discussed in Ref. [26].

Given all of these motivations, we investigate here the collider implications of a gravitino LSP with a charged slepton NLSP with lifetime under (but not much under) a year [27]. In particular, we investigate the possibility of trapping sleptons in material placed just outside Large Hadron Collider (LHC) or International Linear Collider (ILC) detectors. This material may then be moved to some quiet location so that slepton decays may be observed in a relatively background-free environment. Although these objectives may be realized in many ways, we study here the particularly simple possibility of trapping sleptons in water tanks which may be drained periodically to underground reservoirs where the slepton decays may be observed.

In Sec. II] we discuss the relevant properties of sleptons in the gravitino LSP scenario. In Sec. III] we discuss our procedure for maximizing the number of sleptons trapped given a fixed volume of water. This is applied to the cases of the LHC and ILC in Secs. IV] and V. respectively. At the LHC, we find that tens to thousands of sleptons may be trapped each year, depending on the overall mass scale of supersymmetry. At the ILC, typically far fewer sleptons are produced. However, by controlling the beam energy, the velocity of produced sleptons may be tuned to some low value, allowing a large fraction of sleptons to 
be trapped. By exploiting this feature, we find that an order of magnitude more sleptons may be trapped at the ILC than at the LHC. These results imply that percent level studies of slepton decays may be possible. Such studies will have fundamental implications for supergravity, supersymmetry breaking, dark matter, and dark energy. These implications and our conclusions are discussed in Sec. VI

\section{SLEPTON PROPERTIES IN THE GRAVITINO LSP SCENARIO}

\section{A. Slepton Mass}

In supergravity with a gravitino LSP, the slepton NLSP is expected to have a weak-scale mass. Current collider bounds require $m_{\tilde{l}}>99 \mathrm{GeV}$ from null searches for long-lived charged tracks at LEP II [28].

Cosmology brings additional considerations, however. Gravitinos produced in the late decays of sleptons are superWIMP dark matter. Barring the possibility of entropy production after slepton freeze out, the gravitino relic density must therefore satisfy

$$
\Omega_{\tilde{G}} h^{2}=\frac{m_{\tilde{G}}}{m_{\tilde{l}}} \Omega_{\tilde{l}}^{\mathrm{th}} h^{2}<\Omega_{\mathrm{DM}} h^{2}
$$

where $\Omega_{\tilde{l}}^{\text {th }}$ is the slepton's thermal relic density, and the non-baryonic cold dark matter density is constrained to the range $0.094<\Omega_{\mathrm{DM}} h^{2}<0.124$. Assuming $m_{\tilde{G}}$ and $m_{\tilde{l}}$ are not too disparate, this provides an upper bound on the slepton and gravitino masses, since $\Omega_{\tilde{l}}^{\text {th }} \propto m_{\tilde{l}}^{2}$. Without special effects, $\Omega_{\tilde{l}}^{\text {th }} \sim \Omega_{\mathrm{DM}}$ for $m_{\tilde{l}} \sim 700-1000 \mathrm{GeV}$. If $\frac{m_{\tilde{G}}}{m_{\tilde{l}}} \gtrsim 0.1$, the overclosure constraint requires superpartner masses below about $3 \mathrm{TeV}$.

On the other hand, a particularly attractive possibility is that gravitino superWIMPs are most or even all of the non-baryonic dark matter. Although not a strict requirement, one might therefore prefer $\Omega_{\tilde{G}} \approx \Omega_{\mathrm{DM}}$. The decay to the gravitino only reduces the relic density. Without special effects, then, overclosure requires $m_{\tilde{l}} \gtrsim 700-1000 \mathrm{GeV}$. Such heavy sleptons will be difficult to explore at the LHC and are kinematically inaccessible in the first stage of the ILC.

Just as in conventional neutralino dark matter scenarios, however, there are supplementary mechanisms for gravitino production. One such mechanism is co-annihilation. If a neutralino $\chi$ is just slightly heavier than the slepton, it will freeze out with the slepton and later decay to the slepton, adding its thermal relic density to the slepton's. This allows supersymmetric models with much lower slepton masses to produce the correct gravitino superWIMP dark matter density. For example, in minimal supergravity with $A_{0}=0$, $\tan \beta=10$, and $\mu>0$, the desired relic density may be achieved near the $\tilde{\tau}$ LSP $-\chi$ LSP border at $M_{1 / 2}=300 \mathrm{GeV}$, where $m_{\tilde{\tau}} \approx m_{\chi} \approx 120 \mathrm{GeV}[29$ ]. Alternatively, gravitinos may be produced during reheating. For reheating temperatures $T_{\mathrm{RH}} \sim 10^{9} \mathrm{GeV}$, as might be preferred for leptogenesis as discussed above, gravitinos may again be all of the non-baryonic dark matter for slepton masses as low as $120 \mathrm{GeV}$ [12]. It is clear that such effects may be very important. We will consider a variety of slepton masses below, including those within reach of a first stage ILC. We reiterate that all of these considerations depend on the assumption that gravitinos make up all of the dark matter. From a purely particle physics viewpoint, this is clearly optional - some other particle, such as the axion, may be the dark matter. In this case, gravitino LSPs and light NLSP sleptons are perfectly possible without any additional restrictions. 


\section{B. Slepton Lifetime}

The width for the decay of a slepton to a gravitino is

$$
\Gamma(\tilde{l} \rightarrow l \tilde{G})=\frac{1}{48 \pi M_{*}^{2}} \frac{m_{\tilde{l}}^{5}}{m_{\tilde{G}}^{2}}\left[1-\frac{m_{\tilde{G}}^{2}}{m_{\tilde{l}}^{2}}\right]^{4},
$$

assuming the lepton mass is negligible. This decay width depends on only the slepton mass, the gravitino mass, and the Planck mass. In many supersymmetric decays, dynamics brings a dependence on many supersymmetry parameters. In contrast, as decays to the gravitino are gravitational, dynamics is determined by masses, and so no additional parameters enter. In particular, there is no dependence on left-right mixing or flavor mixing in the slepton sector.

\section{Slepton Range in Matter}

Last, it will be crucial to this study to know the range of sleptons in matter. Charged particles passing through matter lose energy by emitting radiation and by ionizing atoms. At lower energies, ionization dominates the energy loss, while at high energies, radiation is the dominant effect. As we will see below, it is unreasonable to expect to stop sleptons with momenta much larger than their rest mass. For the present case, then, ionization losses are dominant, and radiation is negligible.

The average energy loss due to ionization is given by the Bethe-Bloch equation. The low energy approximation to the Bethe-Bloch equation may be derived [30] by first calculating the classical cross section for a collision with fixed impact parameter and energy loss. One then integrates the impact parameter from the Compton wavelength of the free electron as seen by the charged particle to a maximal impact parameter where the particle cannot "see" the electron in the time that it passes by the atom. A more refined treatment yields the Bethe-Bloch equation [31]

$$
\frac{d E}{d x}=K z^{2} \frac{Z}{A} \frac{1}{\beta^{2}}\left[\ln \left(\frac{2 m_{e} c^{2} \beta^{2} \gamma^{2}}{I \sqrt{1+\frac{2 m_{e} \gamma}{M}+\frac{m_{e}^{2}}{M^{2}}}}\right)-\beta^{2}-\frac{\delta}{2}\right],
$$

where $d E / d x$ is the energy loss per $\mathrm{g} \mathrm{cm}^{-2}, K=0.307075 \mathrm{MeV} \mathrm{g}^{-1} \mathrm{~cm}^{2}$, and $m_{e}$ is the electron mass. The material is characterized by its atomic charge $Z$ in units of $e$; its average nucleon number $A$; and its mean ionization energy $I$, which is given for various elements in Ref. 31]. The incoming particle has mass $M$, charge $z$ in units of $e$, velocity $\beta$, and dilation factor $\gamma=\left(1-\beta^{2}\right)^{-1 / 2}$.

The parameter $\delta$ accounts for the fact that incoming particles polarize the surrounding medium. At high energies, this effect may be included by setting

$$
\delta=\Theta\left(E-E_{0}\right)\left[\ln \left(\frac{E^{2}}{M^{2}}-1\right)+\ln \left(\frac{\hbar \omega_{p}}{I}\right)-\frac{1}{2}\right],
$$

where $\omega_{p}$ is the plasma frequency, and $E_{0}$ is the energy at which the effect of polarization is significant. This correction is typically significant for $\beta \gamma \gtrsim 10$. We include this effect, although, as we will see, sleptons that may be trapped in a reasonably sized detector have $\beta \gamma<1$, and so this effect is also irrelevant for the final results of this study. 
TABLE I: Range parameters for lead and water, assuming a $219 \mathrm{GeV}$ slepton.

\begin{tabular}{|c|c|c|c|c|}
\hline Material & $\delta M(\mathrm{MeV})$ & $I(\mathrm{eV})$ & $E_{0}(\mathrm{TeV})$ & $\hbar \omega_{p}(\mathrm{eV})$ \\
\hline Lead & 110 & 820 & 4.4 & 61 \\
\hline Water & 220 & 75 & 1.3 & 21 \\
\hline
\end{tabular}

At low momentum, corrections to Eq. (6) arise from the fact that electrons in matter are bound to atoms. This implies that there is transverse momentum in the collision (Bloch corrections) and that the electron may have momentum comparable to the incident particle (shell corrections). These corrections are significant only when the momentum of the incoming particle is comparable to the electron momentum. As discussed below, we will neglect contributions to the slepton range from very low velocities. For $\mathcal{O}(100) \mathrm{GeV}$ sleptons traveling at the velocities we include, the Bloch and shell corrections may be safely neglected. The Barkas effect, which introduces a dependence on the sign of the charge of the incoming particle is also significant only for very low $\beta$, and may be safely neglected for the velocities we include.

The Bethe-Bloch equation Eq. (6) with $\delta$ as given in Eq. (7) is accurate down to $\beta \sim 0.05$. Below this velocity, experimental data are fit by parameterization schemes. We do not have this luxury because, of course, there are as yet no experimental data for sleptons. Although we could use the parameterizations adopted for standard model particles, it has been found that the heavier the particle the worse these models are at describing the low momentum behavior [32].

Rather than grapple with how sleptons should behave at $\beta<0.05$, we adopt the following procedure for determining the slepton range in matter. For a certain low velocity, $d E / d x$ in Eq. (6) peaks and then rapidly drops to zero. We denote this velocity $\beta_{\text {peak }}$; its value is typically $\sim 0.01$. At $\beta_{\text {peak }}$, the value of $d E / d x$ is very high, and we expect that the distance the slepton travels as it slows from $\beta_{\text {peak }}$ to thermal equilibrium is negligible relative to the distance it traveled in slowing down from its initial velocity to $\beta_{\text {peak. }}$. We further take the continuous slowing down approximation. With these assumptions, the range $R$ in $\mathrm{g} \mathrm{cm}^{-2}$ for a slepton with energy $E^{\prime}$ is

$$
R\left(E^{\prime}\right)=\frac{1}{K} \frac{A}{Z} \int_{M+\delta M}^{E^{\prime}} d E \frac{\frac{M^{2}}{E^{2}}-1}{\ln \left(\frac{2 m_{e} c^{2}}{I} \frac{E^{2}-M^{2}}{\sqrt{M^{2}+2 E m_{e}+m_{e}^{2}}}\right)+\left(\frac{M^{2}}{E^{2}}-1\right)-\delta},
$$

where $\delta$ is given in Eq. (7), and $M+\delta M$ is the energy at which the slepton has velocity $\beta_{\text {peak }}$. The values used for $\delta M, I, E_{0}$, and $\hbar \omega_{p}$ for the particular case of a $219 \mathrm{GeV}$ slepton are given in Table

The Bethe-Bloch equation gives an average value of the stopping power, but there are always fluctuations about these values. For thin materials the most probable energy loss may be very different from the mean energy loss [31]. However, if the path length in material is large enough, the energy loss distribution is Gaussian. The criterion for Gaussianity has been found in Ref. [33] to be

$$
\kappa=\frac{\frac{K}{2} \frac{Z}{A} \frac{1}{\beta^{2}} \rho x}{T}>10
$$

where

$$
T=\frac{2 m_{e} c^{2} \beta^{2} \gamma^{2}}{\sqrt{1+\frac{2 m_{e} \gamma}{M}+\frac{m_{e}^{2}}{M^{2}}}}
$$



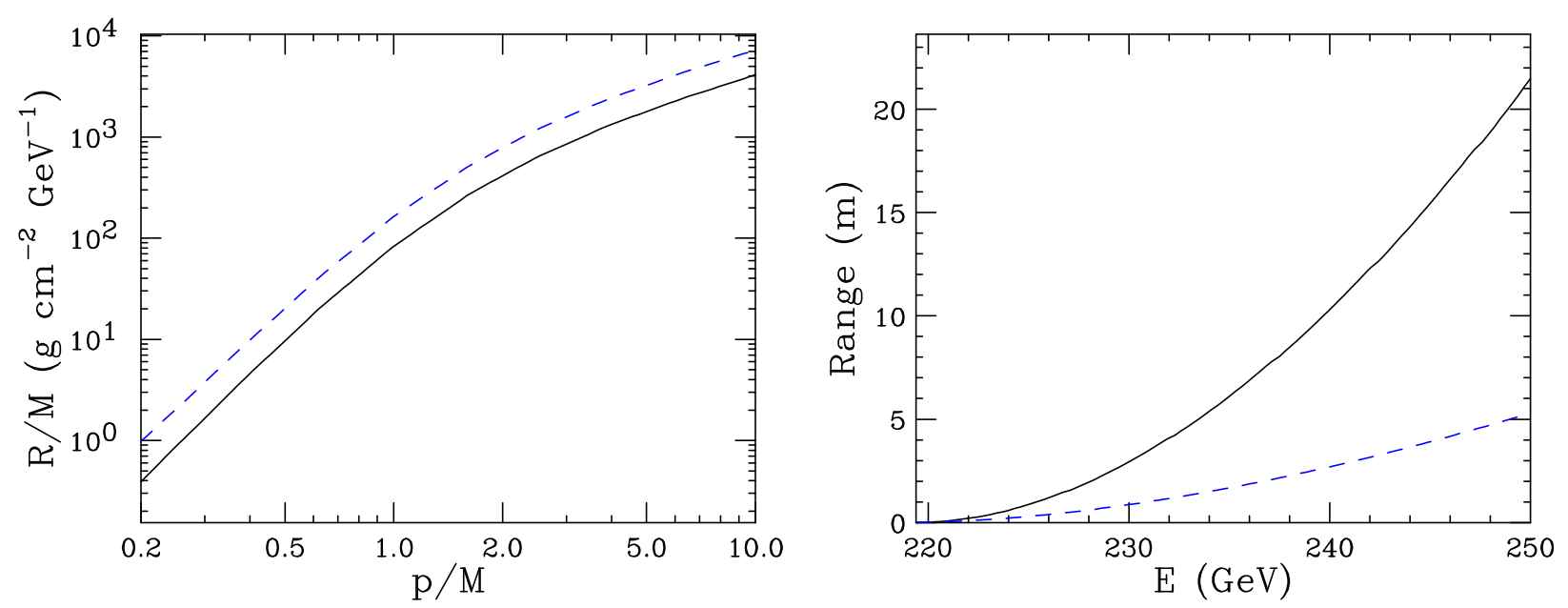

FIG. 1: The range to mass ratio $R / M$ as a function of $p / M=\beta \gamma$ (left), and, for the specific case of a slepton with mass $219 \mathrm{GeV}$, the range as a function of energy (right). Results are given for water (solid) and lead (dashed).

and $x$ is the path length. For LHC and ILC detectors, $\kappa \gtrsim \mathcal{O}(100)$. Given that the energy distribution of produced sleptons is not wildly fluctuating, inclusion of this Gaussian range distribution will have a negligible impact on our results, and we do not include it.

Using the procedure described above, the range to mass ratios $R / M$ for lead and water are given in Fig. 1. These results agree beautifully with published results [31]. The range as a function of energy for the particular case of a slepton with mass $219 \mathrm{GeV}$ is also given. A slepton with this mass and energy $240 \mathrm{GeV}(250 \mathrm{GeV})$ travels about 10 meters (21 meters) in water before stopping.

\section{SLEPTON TRAP OPTIMIZATION}

We will consider the possibility of trapping sleptons in water placed just outside a collider detector. Of course, any material may be used, and our analysis, at the level we have pursued it, is valid for any material. We consider water to be promising, however, as it is potentially feasible to increase the concentration of sleptons and/or move it to a place where slepton decays may be observed in a background-free environment.

We would like to optimize the placement and shape of the water tank. If the gravitino LSP scenario is realized in nature, given the implications described in Sec. VI, we consider the importance of slepton trapping studies to be sufficient to enlarge detector halls. We therefore do not consider constraints from existing detector halls in considering trap geometries. Even with this simplification, however, a detailed discussion of optimization requires careful accounting of the various LHC and ILC detector component geometries, costs, and other factors.

Rather than undertake such a detailed study, we consider here a simple detector model to highlight the physics that may be explored in more detail in following studies. We characterize the properties of the inner detector by two parameters: $r_{\text {in }}$, the distance from the interaction point (IP) to the outside of the detector, and $r_{\text {in }}^{\text {we }}=\int \rho d l$, the densityweighted distance between the IP and the outside of the detector, typically measured in 


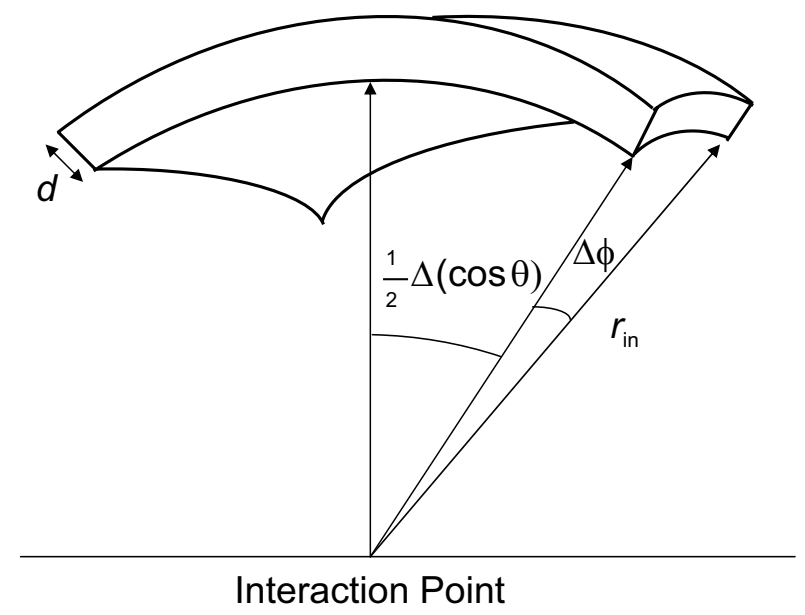

FIG. 2: Diagram of the slepton trap geometry. The trap is assumed to be a spherical shell with inner radius $r_{\mathrm{in}}$, and depth $d$ as shown. The angular parameters $\frac{1}{2} \Delta(\cos \theta)$ and $\Delta \phi$ of Eq. (11) are also indicated.

meters water equivalent (mwe). We will assume that $r_{\text {in }}$ and $r_{\text {in }}^{\text {we }}$ are independent of polar angle $\theta$ and azimuthal angle $\phi$; that is, we model the inner detector as spherically symmetric in both size and material depth.

The amount of energy lost by a slepton traveling through a detector is determined by $r_{\text {in }}^{\text {we }}$. (For realistic detector sizes, the tracks of sleptons with sufficient energy to pass through the detector have negligible curvature.) The radius of the LHC detectors is approximately $12 \lambda_{I}$ in the direction perpendicular to the beam line, where $\lambda_{I}$ is the nuclear interaction length [34, 35]. This number depends on rapidity, but we take this minimal value for our spherical detector. The ILC detector is expected to be slightly smaller, but sheets of lead or other material can always be used to increase the effective radius to that of the LHC detectors. As we will see, this may be advantageous. Given these considerations, we assume for this study that the energy loss at the LHC and ILC is approximately that of a particle traveling through $12 \lambda_{I}$ of water. Since $\lambda_{I}=83.6 \mathrm{~g} \mathrm{~cm}^{-2}$ for liquid water, this sets $r_{\mathrm{in}}^{\mathrm{we}}=10$ mwe.

The number of trapped sleptons is, of course, maximized by placing the water tank as close to the IP as possible. ${ }^{1}$ In addition, as we will see, the polar angle distribution of slow sleptons, that is, those that have a chance of being trapped, is either uniform or peaked perpendicular to the beam line at polar angle $\theta=\pi / 2$. To optimize the water tank placement, then, we consider the family of tank geometries specified by

$$
\begin{aligned}
r_{\text {in }}<\quad & <r_{\text {in }}+d \\
|\cos \theta| & <\frac{1}{2} \Delta(\cos \theta) \\
0<\phi & <\Delta \phi,
\end{aligned}
$$

where $(r, \theta, \phi)$ are polar coordinates centered on the IP, and $d$ is the tank's radial depth.

\footnotetext{
${ }^{1}$ This assumes that no additional material is added between the detector and the trap. It may be advantageous to place the trap farther away if a material that slows sleptons is added between the detector and the trap. An example is discussed in Sec. $\mathrm{V}$
} 


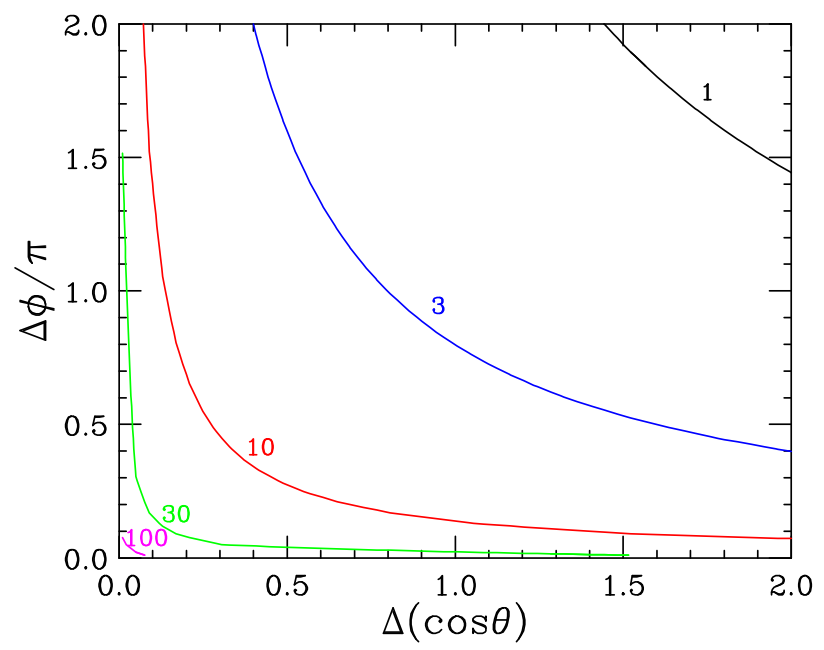

FIG. 3: The depth $d$ in meters of a 1 kton water trap in the $(\Delta(\cos \theta), \Delta \phi)$ plane for $r_{\text {in }}=10 \mathrm{~m}$.

This geometry is illustrated in Fig. 2. All sleptons with range less than $r_{\text {in }}^{\text {we }}$ are trapped in the detector. However, all sleptons with range between $r_{\text {in }}^{\text {we }}$ and $r_{\text {in }}^{\text {we }}+d$, and polar and azimuthal angles in the ranges given in Eq. (11) are caught in the water tank. ${ }^{2}$ The water tank volume is

$$
V=\frac{1}{3}\left[\left(r_{\text {in }}+d\right)^{3}-r_{\text {in }}^{3}\right] \Delta(\cos \theta) \Delta \phi .
$$

In summary, the number of trapped sleptons is

$$
N\left(V, \Delta(\cos \theta), \Delta \phi, r_{\mathrm{in}}, r_{\mathrm{in}}^{\mathrm{we}}\right),
$$

where the first four parameters determine the depth $d$ through Eq. (12). Sample depths for a 1 kton trap $\left(V=1000 \mathrm{~m}^{3} \mathrm{we}\right)$ are shown in Fig. 3. In the following sections, we fix $r_{\text {in }}$ and $r_{\text {in }}^{\text {we }}$ to appropriate values and choose three representative sizes $V$. We then scan over all possible values of $\Delta(\cos \theta)$ and $\Delta \phi$ to maximize $N$. In this way, we determine the optimal shape for the water tank and the maximal number of sleptons that may be trapped.

\section{SLEPTON TRAPPING AT THE LHC}

The LHC is scheduled to collide protons with protons at $\sqrt{s}=14 \mathrm{TeV}$ beginning in 2007-08. Its initial luminosity is expected to be $10 \mathrm{fb}^{-1} / \mathrm{yr}$, growing to $100 \mathrm{fb}^{-1} / \mathrm{yr}$. We present results below for $100 \mathrm{fb}^{-1}$, a high luminosity year.

The ATLAS and CMS detectors are cylindrical with radii $10 \mathrm{~m}$ and $8 \mathrm{~m}$, respectively. As explained in Sec. III. we choose $r_{\text {in }}=10 \mathrm{~m}$ and $r_{\text {in }}^{\text {we }}=10$ mwe. Although we consider only ATLAS and CMS, other detectors could provide promising opportunities. For example, $\mathrm{LHCb}$ is an asymmetric detector and allows for smaller values of $r_{\mathrm{in}}^{\text {we }}$ and $r_{\text {in }}$. This may make improved results possible and is a possibility well worth considering.

In the gravitino LSP scenario, all supersymmetry events produce two long-lived NLSP sleptons. The dominant source of NLSPs at hadron colliders is typically pair production

\footnotetext{
${ }^{2}$ In practice, for the Monte-Carlo simulations below, we smooth distributions in $\phi$ by including all events that pass the $r$ and $\cos \theta$ cuts with weight $\Delta \phi / 2 \pi$.
} 


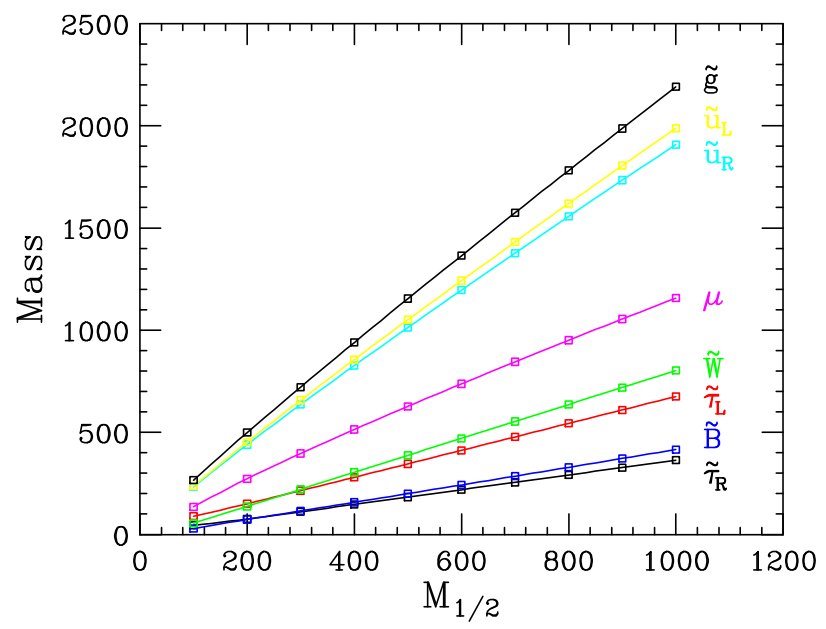

FIG. 4: Representative superpartner masses as a function of $M_{1 / 2}$ in minimal supergravity with fixed $m_{0}=0, A_{0}=0, \tan \beta=10$, and $\mu>0$. The supersymmetry parameter $\mu$, which governs the Higgsino masses, is also shown.

of strongly interacting superpartners. The number of trapped sleptons is therefore modeldependent in a complicated way, as it depends not only on the slepton mass but also sensitively on the masses of colored superpartners and their cascade decay patterns.

Here we consider minimal supergravity with the following parameters:

$$
m_{0}=0, \quad M_{1 / 2}=300-900 \mathrm{GeV}, \quad A_{0}=0, \quad \tan \beta=10, \quad \mu>0 .
$$

When the gravitino is not the LSP, this is in the excluded "stau LSP" region. In the present scenario with a gravitino LSP, however, these models are allowed, and this one-dimensional family of models provides a simple set with which we can explore the prospects for trapping sleptons at the LHC. The lower bound on $M_{1 / 2}$ is determined by the requirement of a stau NLSP. The number of trapped staus rapidly diminishes as $M_{1 / 2}$ increases, and the upper bound on $M_{1 / 2}$ is roughly where only a few staus may be trapped per year. The superpartner spectra for various $M_{1 / 2}$ in these models are given in Fig. 4.

The mass spectra of Fig. 4 are calculated by ISASUSY 7.69 with top mass $m_{t}=$ $175 \mathrm{GeV}$ [36]. We have also used this package to generate LHC events. 100,000 nonstandard model events were generated for minimal supergravity models with the parameters given above and $M_{1 / 2}$ varying from $300 \mathrm{GeV}$ to $900 \mathrm{GeV}$ in $100 \mathrm{GeV}$ increments. Helicity correlations are not included in ISAJET. However, there are typically several steps in decay chains leading to the NLSP, and so we do not expect helicity correlations to have a significant impact on the NLSP distributions or on our final results.

In Fig. 5. we show the energy distribution of NLSP staus for the $M_{1 / 2}=600 \mathrm{GeV}$. Although many staus are produced, most of these are extremely energetic and impossible to stop in a reasonable distance. Of course, a trap could be set up far from the IP so that the intervening earth slows down the slepton, but such a trap would be too far away to have a reasonable solid angle coverage for any realistic volume $V$.

The $\cos \theta$ distribution at $M_{1 / 2}=600 \mathrm{GeV}$ is shown in Fig. 6. Given all produced NLSPs, the distribution is strongly peaked in the beam directions. However, imposing a cut on energy, we find that the slow staus are produced roughly isotropically. Thus, for those staus that we might reasonably hope to be trapped, any polar angle is as good as any other. Given 


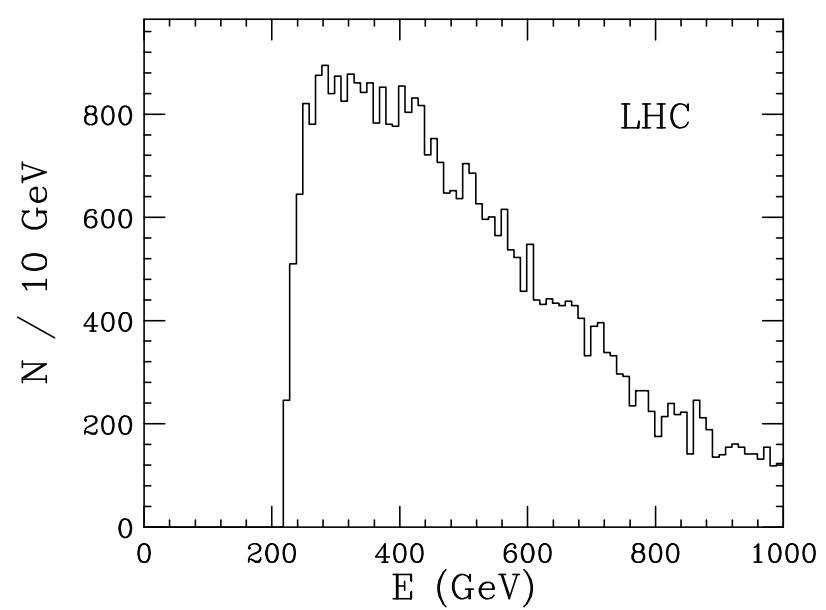

FIG. 5: The energy distribution of NLSP staus produced at the LHC for integrated luminosity $100 \mathrm{fb}^{-1}$ and minimal supergravity with $m_{0}=0, M_{1 / 2}=600 \mathrm{GeV}, A_{0}=0, \tan \beta=10$, and $\mu>0$. The NLSP stau mass is $219 \mathrm{GeV}$.

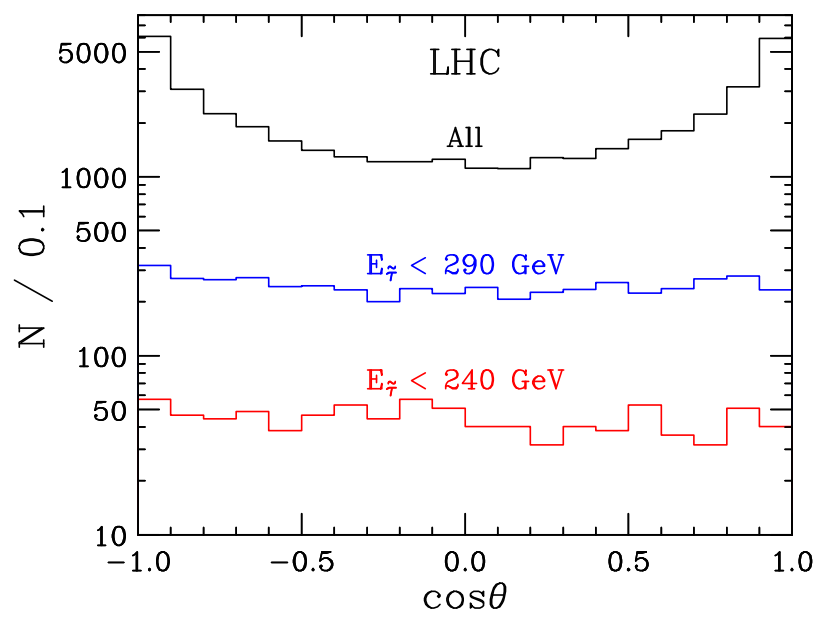

FIG. 6: The $\cos \theta$ distribution of NLSP staus produced at the LHC for integrated luminosity $100 \mathrm{fb}^{-1}$ and minimal supergravity with $m_{0}=0, M_{1 / 2}=600 \mathrm{GeV}, A_{0}=0, \tan \beta=10, \mu>0$. The NLSP stau mass is $219 \mathrm{GeV}$. Distributions are given for all staus, $E_{\tilde{\tau}}<290 \mathrm{GeV}(R<100$ mwe), and $E_{\tilde{\tau}}<240 \mathrm{GeV}(R<10$ mwe). The total distribution is strongly peaked along the beam directions, but slow sleptons are produced isotropically.

the reality that LHC detectors are cylindrical, however, the closest a trap may be placed to the IP is at $\cos \theta=0$, justifying our choice of centering our trap geometries at $\cos \theta=0$, as parameterized in Eq. (11).

The number of trapped sleptons for optimized trap shape and placement and various trap volumes is given in Fig. [7. The trap is optimized as described in Sec. III] we scan over all possible $\Delta(\cos \theta)$ and $\Delta \phi$, and find the combination that maximizes the number of sleptons that stop in the trap.

We find that only a small fraction of produced sleptons can be trapped. For example, for $M_{1 / 2}=600 \mathrm{GeV}, 4.2 \times 10^{4}$ NLSP sleptons are produced, but only 260, 40, and 9 are trapped in water tanks of size 10, 1 , and 0.1 kton, respectively. For all models considered, 


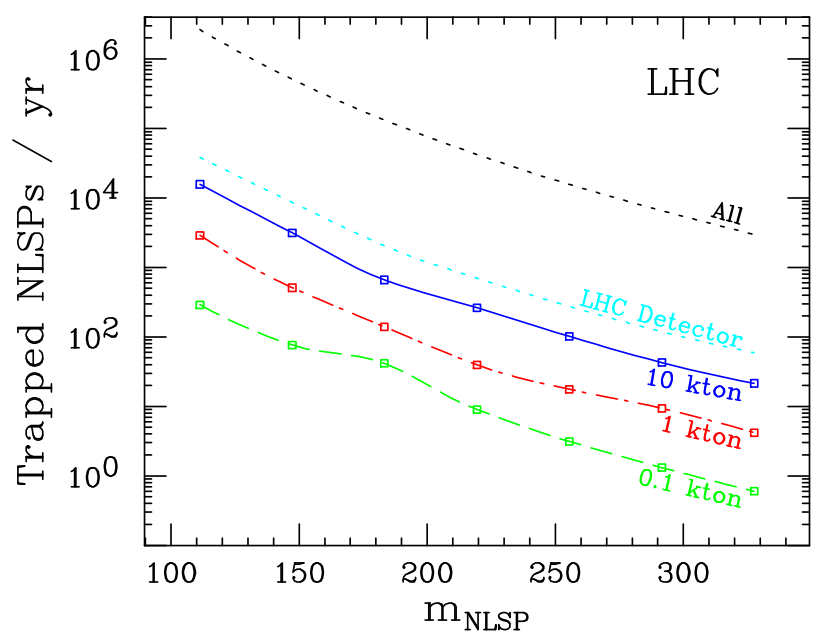

FIG. 7: The number of sleptons trapped per year at the LHC in water tanks of size 10 kton (solid), 1 kton (dot-dashed), and 0.1 kton (dashed). The total number of sleptons produced is also shown (upper dotted), along with the number of sleptons trapped in the LHC detector (lower dotted). The water tank shape and placement have been optimized as described in the text. These results assume luminosity $100 \mathrm{fb}^{-1} / \mathrm{yr}, r_{\text {in }}=10 \mathrm{~m}, r_{\text {in }}^{\text {we }}=10$ mwe, and minimal supergravity models with $M_{1 / 2}=300,400, \ldots, 900 \mathrm{GeV}, m_{0}=0, A_{0}=0, \tan \beta=10$, and $\mu>0$.

the 10 kton trap is optimized for $\Delta(\cos \theta)=\Delta \phi / \pi=2$; for this large volume, the trap is sufficiently deep that there is little be gained by making the trap deeper at the expense of solid angle coverage. For $V=1$ kton, the number of trapped sleptons is optimized by traps with less-than-maximal angular coverage and depths of $d \approx 4 \mathrm{~m}$. Note that, because slow sleptons are produced isotropically, the number of trapped sleptons depends, to a good approximation, on $\Delta(\cos \theta)$ and $\Delta \phi$ only through their product $\Delta(\cos \theta) \Delta \phi$.

Despite the low efficiency for trapping, so many NLSPs are produced at the LHC that significant numbers of NLSPs may still be trapped. As anticipated, the results are heavily dependent on the overall scale of superpartner masses. For $V=10 \mathrm{kton}$ and $m_{\mathrm{NLSP}}=$ $100-300 \mathrm{GeV}$, the number of trapped sleptons varies from $\mathcal{O}\left(10^{4}\right)$ to $\mathcal{O}(10)$. For the lighter sleptons considered, these results imply that sufficient numbers of sleptons may be trapped to do precision studies of slepton decay properties. Assuming that slepton decays may be observed in a background-free environment, we expect percent level measurements of slepton decay widths.

Note that for all $M_{1 / 2}$, a larger number of sleptons range out in our spherically symmetric LHC detector than can be trapped in even the 10 kton water trap. For $M_{1 / 2}=600 \mathrm{GeV}$, 700 are trapped in the LHC detector itself. The LHC detector has a large volume and benefits from the fact that it begins at the IP and so has a large angular coverage without sacrificing depth. Unfortunately, it is not clear to what extent these sleptons may be used their decays are out of time, occur away from the IP, and take place in an environment with significant cosmic ray background. Given the large number of sleptons that are automatically trapped in the LHC detector itself, however, it is certainly worthwhile to explore ways to exploit them. 


\section{SLEPTON TRAPPING AT THE ILC}

In its first stage, the ILC will collide electrons and positrons with center of mass energies up to $500 \mathrm{GeV}$. In this first stage, the luminosity has been estimated to be $340 \mathrm{fb}^{-1} / \mathrm{yr}$ for the TESLA design [37] and $220 \mathrm{fb}^{-1} / \mathrm{yr}$ for the NLC/JLC [38]. For this study, we assume luminosity $300 \mathrm{fb}^{-1} / \mathrm{yr}$. As in the LHC analysis above, we present results for one year of running.

At present ILC detectors are expected to be slightly smaller than their LHC counterparts. To be conservative, we assume $r_{\text {in }}=10 \mathrm{~m}$ and $r_{\text {in }}^{\text {we }}=10$ mwe, the same parameters we assumed in the LHC case. Of course, if the detector is smaller than this, it can always be supplemented by adding plates of lead, for example, to mock up these parameters. As we will see, in the ILC case, such an approach may in fact enhance our results very significantly.

At the ILC, scanning over supersymmetry models with a broad range of superpartner mass scales, as done in the LHC analysis above, is not particularly informative. Models with heavy superpartners are simply out of reach, and no sleptons may be produced, much less trapped. On the other hand, for models with superpartners within reach, the ILC beam energy may be tuned to optimize the number of trapped sleptons, to some extent offsetting variations in the scale of superpartner masses in these models. As we will see, the crucial feature is not the exact mass of the slepton NLSP, but rather the presence of other nearly degenerate superpartner states.

For the ILC, then, we limit our analysis to two models. In the first, which we denote "NLSP only," the only superpartner within reach of the ILC is an NLSP $\tilde{\tau}_{R}$ with mass 219 $\mathrm{GeV}$. This is representative of the minimal case where the gravitino LSP scenario may be probed at the ILC. Of course, in many realistic models, there are a number of other superpartners, notably other sleptons, fairly degenerate with the NLSP. We therefore consider also a second model, which we denote "mSUGRA," which is minimal supergravity with $M_{1 / 2}=600 \mathrm{GeV}, A_{0}=0, \tan \beta=10$, and $\mu>0$. This model contains not only the $219 \mathrm{GeV}$ $\tilde{\tau}_{R}$ of the "NLSP only" model, but also right-handed selectrons, right-handed smuons, and a neutralino within the kinematic reach of a $500 \mathrm{GeV}$ ILC. The mSUGRA model is one of the family of models considered previously in the LHC analysis, allowing us to compare the LHC and ILC at one particular model point. Because it contains the "NLSP only" model as a subset, it also allows us to see the effect of having other accessible and fairly degenerate superpartners. The accessible standard model superpartners of the two models and their masses are:

$$
\left.\begin{array}{rl}
m_{\chi} & 242.9 \mathrm{GeV} \\
m_{\tilde{e}_{R}}, m_{\tilde{\mu}_{R}} & 227.2 \mathrm{GeV} \\
m_{\tilde{\tau}_{R}} & 219.3 \mathrm{GeV} \quad\} \text { NLSP only }
\end{array}\right\} \text { mSUGRA }
$$

We generate $10^{4}$ non-standard model ILC events for the mSUGRA model with ISASUSY 7.69 with $m_{t}=175 \mathrm{GeV}$ [36]. Events for the "NLSP only" model are compiled by selecting the prompt stau events from this event sample. We choose beam width $0.12 \mathrm{~mm}$, and beamstrahlung parameter $\Upsilon=0.1072$, and allow the subprocess energy to vary over the entire range from $2 m_{\mathrm{NLSP}}$ to $\sqrt{s}$.

In the "NLSP only" model, the NLSP staus are produced through $e^{+} e^{-} \rightarrow \gamma, Z \rightarrow$ $\tilde{\tau}^{+} \tilde{\tau}^{-}$. The stau energy distribution is therefore given by the beam energy modified by initial state radiation (ISR) and beamstrahlung. An example with $\sqrt{s}=500 \mathrm{GeV}$ is given in Fig. 8. The stau polar angle distribution is also given in Fig. 8. Despite ISR and beamstrahlung, it retains the $\sin ^{2} \theta$ shape of the parton-level process. The best place to 

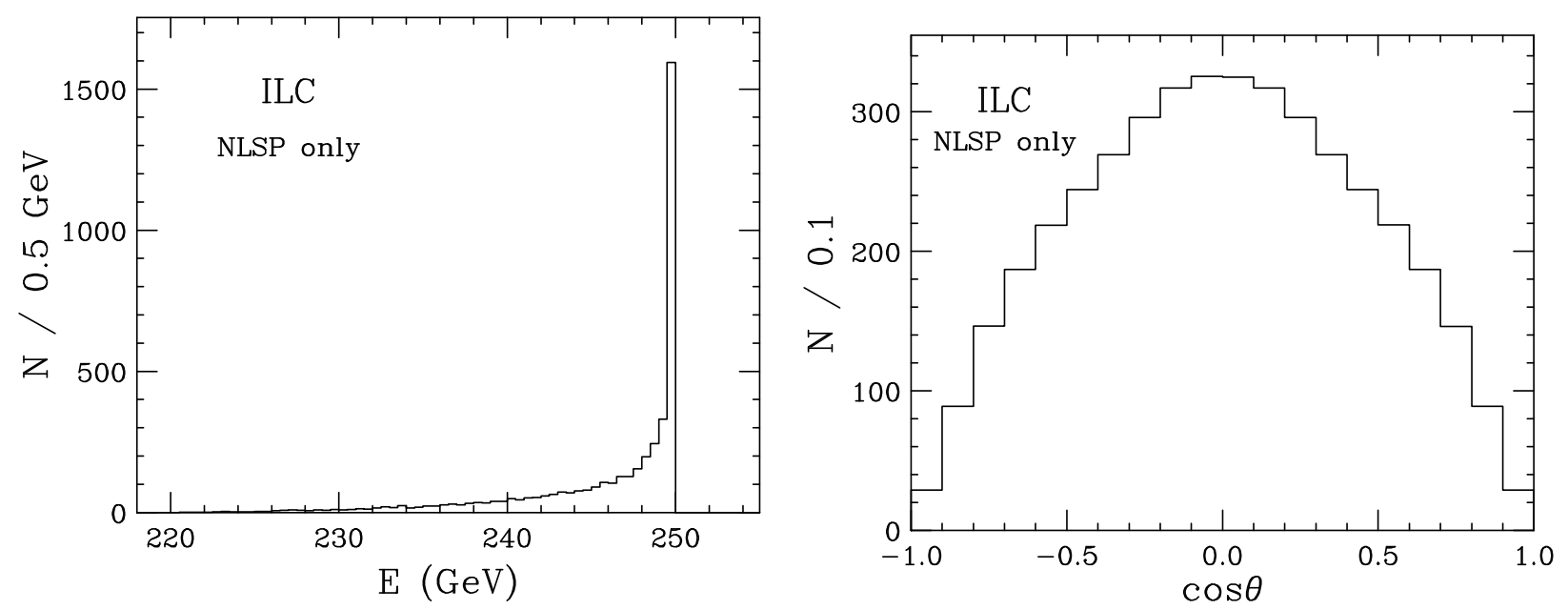

FIG. 8: Energy (left) and $\cos \theta$ (right) distributions for NLSP staus produced at the ILC assuming the "NLSP only" model, in which the only accessible superpartner is the NLSP stau with mass $219 \mathrm{GeV}$. Results are for $\sqrt{s}=500 \mathrm{GeV}$ and integrated luminosity $300 \mathrm{fb}^{-1}$.

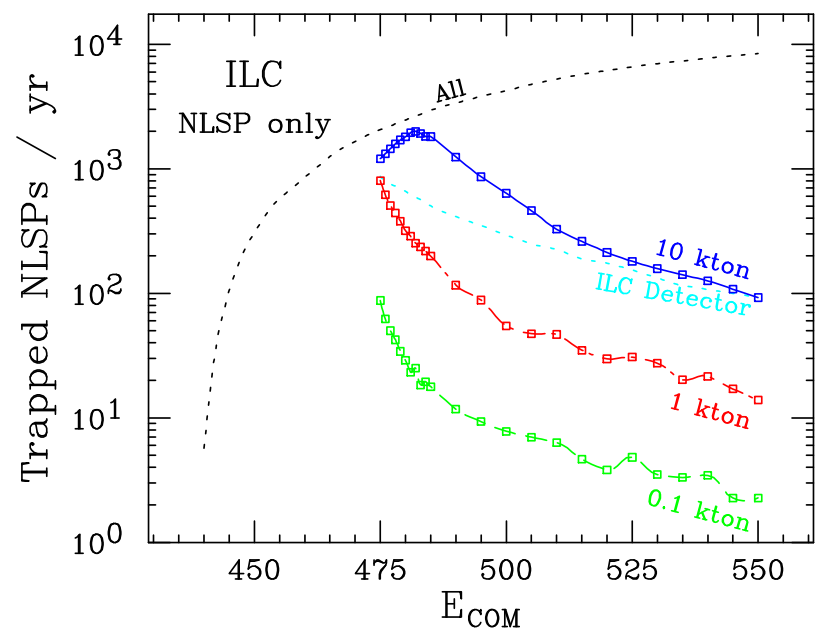

FIG. 9: The number of sleptons trapped per year at the ILC in 10 kton (solid), 1 kton (dotdashed), and 0.1 kton (dashed) water traps. The total number of sleptons produced is also shown (upper dotted) along with the number of sleptons trapped in the ILC detector (lower dotted). The trap shape and placement have been optimized, and we assume $r_{\mathrm{in}}=10 \mathrm{~m}$ and $r_{\text {in }}^{\text {we }}=10$ mwe, luminosity $300 \mathrm{fb}^{-1} / \mathrm{yr}$ and the NLSP only model, where the only accessible superpartner is a 219 GeV NLSP stau.

trap sleptons is therefore perpendicular to the beam line, justifying our choice of centering our trap geometries at $\cos \theta=0$, as parameterized in Eq. (11).

The number of trapped sleptons for various trap sizes as a function of center-of-mass energy $\sqrt{s}$ is given in Fig. 9. For $\sqrt{s}<475 \mathrm{GeV}$, no staus escape the ILC detector. At $\sqrt{s}=475 \mathrm{GeV}$, however, sleptons in the sharp peak of the energy distribution escape the ILC detector and may be caught in a fairly thin water tank placed just outside the ILC detector.

As evident in Fig. 9, for the 1 and 0.1 kton water traps, the number of trapped staus is 


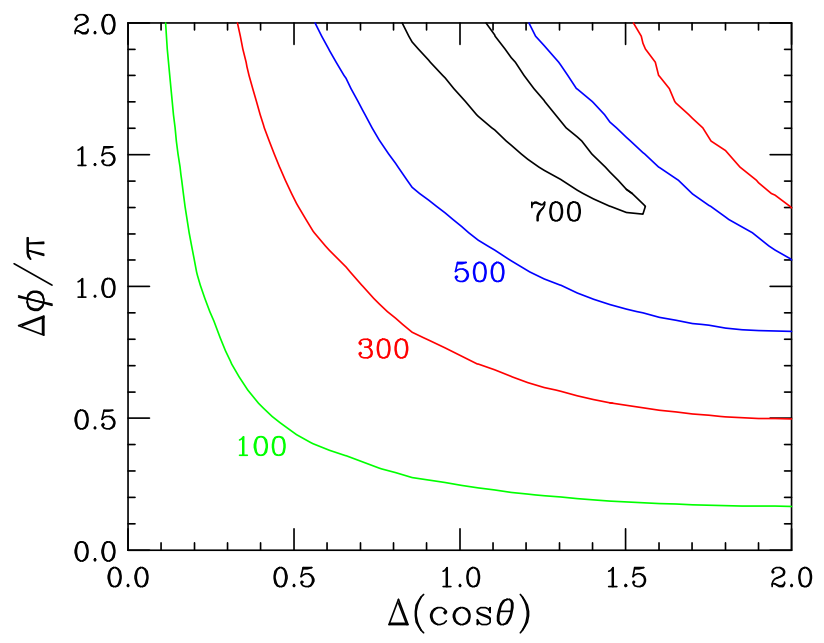

FIG. 10: The number of trapped staus at the ILC in the $(\Delta(\cos \theta), \Delta \phi)$ plane for the "NLSP only" model, $\sqrt{s}=475 \mathrm{GeV}$, and integrated luminosity $300 \mathrm{fb}^{-1} / \mathrm{yr}$.

maximized when the beam energy is tuned to produce staus that just barely emerge from the ILC detector. The dependence on trap parameters is illustrated in Fig. 10, The optimized trap configuration has $\Delta(\cos \theta) \approx 1$ and $\Delta \phi=2 \pi$; because the stau distribution is peaked at $\cos \theta=0$, it is beneficial to sacrifice coverage at high rapidity to make the trap deeper. For a 10 kton trap, the trap is sufficiently thick that the best results are achieved for slightly higher beam energies where more of the ISR/beamstrahlung tail may be caught. For a 10 kton trap, we find that the optimal trap configuration has $\Delta(\cos \theta) \approx 2$ and $\Delta \phi=2 \pi$.

For all trap sizes, however, the number of trapped sleptons is maximized for beam energies near the $475 \mathrm{GeV}$ threshold. The tunable beam energy and well-defined initial state are wellknown virtues of the ILC, but these are exploited in a qualitatively novel way here to produce slow NLSP sleptons that may be easily caught. Relative to the case at the LHC, a much larger fraction of the produced staus can be caught. For example, of the 2650 staus produced at $\sqrt{s}=482 \mathrm{GeV}$ in a year, 2000 staus may be trapped in an optimized 10 kton water trap! Such results imply promising prospects for slepton trapping even in the minimal case when the only superpartner accessible at the ILC is the NLSP slepton.

Before considering the mSUGRA model, we note that the number of produced staus continues to rise well beyond its value at $\sqrt{s}=475 \mathrm{GeV}$. This suggests that our results may be improved significantly by placing some dense material between the ILC detector and the water tank. By adding material depth to the ILC detector, the threshold at which sleptons just barely emerge is moved to higher $\sqrt{s}$ where the stau pair production cross section is higher. For a dense material, such as lead, this can be achieved without increasing $r_{\text {in }}$ much. Such a strategy may in any case be required to smooth out variations in $r_{\text {in }}^{\text {we }}$ inherent in realistic detectors. Although we have not investigated this in detail, we expect that a large enhancement may be possible.

We now turn to the mSUGRA model. In this model, NLSP staus may again be produced directly, but now they may also be produced in several other ways: first, by $e^{+} e^{-} \rightarrow \tilde{e}^{+} \tilde{e}^{-}, \tilde{\mu}^{+} \tilde{\mu}^{-}$followed by $\tilde{e} \rightarrow e \tilde{\tau} \tau$ and $\tilde{\mu} \rightarrow \mu \tilde{\tau} \tau$, and second, by $e^{+} e^{-} \rightarrow \chi \chi$, followed by $\chi \rightarrow \tilde{\tau} \tau$, or through the cascade $\chi \rightarrow \tilde{e}, \tilde{\mu} \rightarrow \tilde{\tau}$. The energy and $\cos \theta$ distributions 

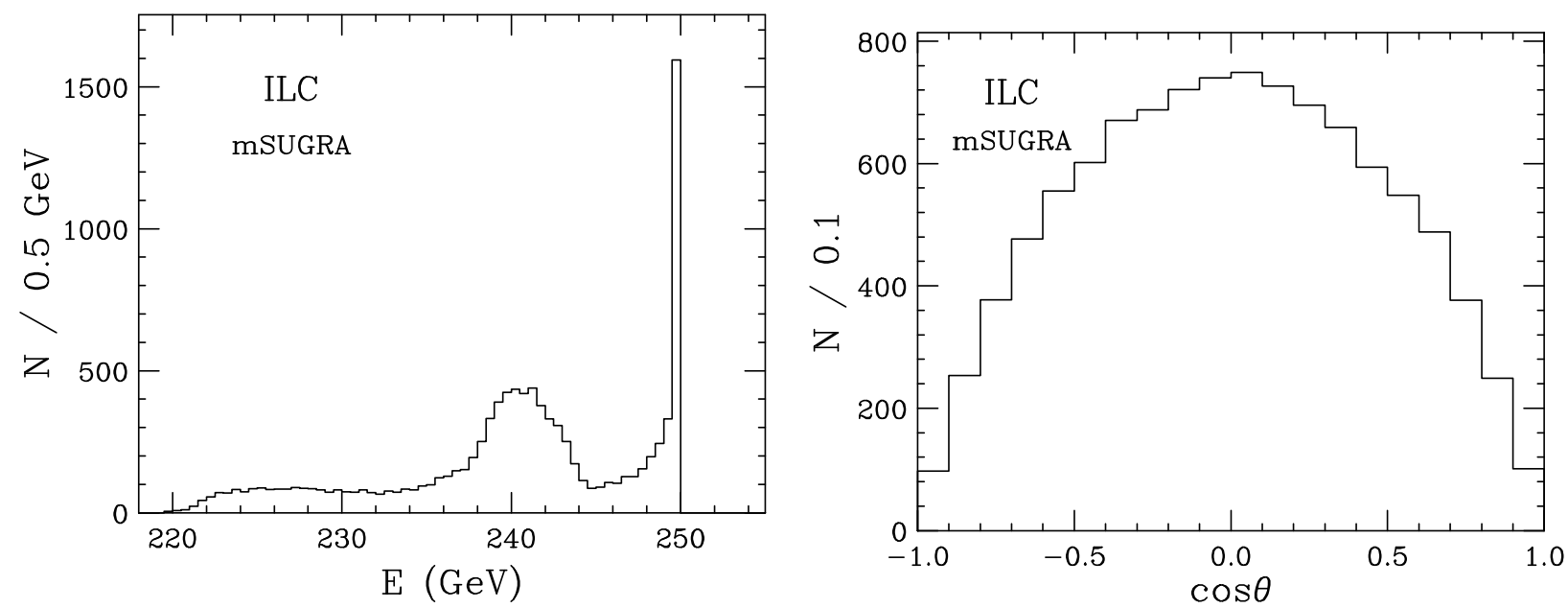

FIG. 11: Energy (left) and $\cos \theta$ (right) distributions for NLSP staus produced at the ILC in the mSUGRA model with $m_{0}=0, M_{1 / 2}=600 \mathrm{GeV}, A_{0}=0, \tan \beta=10, \mu>0$. Results are for $\sqrt{s}=500 \mathrm{GeV}$ and integrated luminosity $300 \mathrm{fb}^{-1} / \mathrm{yr}$.

of NLSP staus in the mSUGRA model are shown in Fig. 11. ${ }^{3}$ As evident in Fig. 11, the additional sources of staus increase the total number of staus significantly, but just as significant, the cascade decays produce a broad and flat tail in the energy distribution extending nearly down to $m_{\tilde{\tau}}$. The $\cos \theta$ distribution is nevertheless still peaked at $\cos \theta=0$.

The number of trapped staus per year for the mSUGRA model are given in Fig. 12. The presence of additional accessible superpartner states has a significant impact — for all trap sizes considered, large numbers of staus may be trapped even for beam energies well above $475 \mathrm{GeV}$. This is a consequence of the broad energy distribution of NLSP staus, which in turn follows from the existence of other fairly degenerate superpartners.

In the mSUGRA case, we may also compare these ILC results directly with results from the LHC analysis given above. The LHC results for $M_{1 / 2}=600 \mathrm{GeV}$ from Sec. IV are given by the lines marked "LHC" in Fig. 12, Comparing results of similarly-sized water traps, we find that for this particular model, the ILC will be able to trap a factor of $\sim 10$ more staus than the LHC. Again, for the reasons discussed above, a significant enhancement of these ILC results may be possible if one considers inserting lead between the ILC detector and the water trap, and as many as $\mathcal{O}\left(10^{4}\right)$ staus may be trapped by reasonably sized water traps using this more general approach.

\section{IMPLICATIONS AND CONCLUSIONS}

Although the implications of supergravity for cosmology and particle physics have been considered in great detail for decades, most work has been centered on scenarios in which the LSP is a standard model superpartner. Here we have explored the gravitino LSP scenario. Recent work has found significant cosmological motivations for this possibility, as

\footnotetext{
${ }^{3}$ Helicity correlations between production and decay are not included in our event generation. These are, of course, absent for scalar particles, but may modify both the energy and $\cos \theta$ distributions for staus produced through $\chi$ pair production.
} 


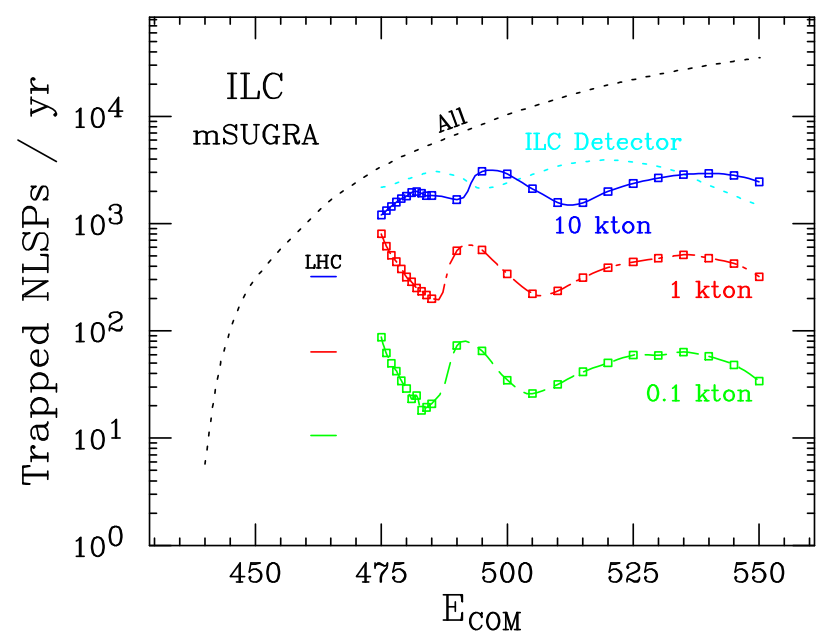

FIG. 12: The number of sleptons trapped per year at the ILC in 10 kton (solid), 1 kton (dot-dashed), and 0.1 kton (dashed) water traps. The total number of sleptons produced is also shown (upper dotted) along with the number of sleptons trapped in the ILC detector (lower dotted). The trap shape and placement have been optimized, and we assume $r_{\text {in }}=10 \mathrm{~m}$ and $r_{\text {in }}^{\text {we }}=10$ mwe, luminosity $300 \mathrm{fb}^{-1} / \mathrm{yr}$ and the mSUGRA model with $m_{0}=0, M_{1 / 2}=600 \mathrm{GeV}, A_{0}=0, \tan \beta=10, \mu>0$. For comparison, the number of trapped staus at the LHC for various trap volumes is also shown.

the gravitino may explain dark matter, and the scenario may resolve current difficulties in Big Bang nucleosynthesis and with leptogenesis. We considered here a novel implication for collider physics, namely that NLSP sleptons may be collected in water traps before their decays to the gravitino. These sleptons may then be concentrated and transported to some quiet environment where their decays may be studied in detail.

By optimizing the water trap shape and placement and considering a variety of sizes, we have first explored the prospects for trapping sleptons at the LHC. The number that may be trapped is highly model-dependent. For minimal supergravity with $m_{0}=0$, we find that as many as $10^{4}$ staus may be stopped in a $10 \mathrm{kton}$ trap when the sleptons have mass around $100 \mathrm{GeV}$. This is as light as is allowed by current bounds. For a less optimistic scenario, say, with $219 \mathrm{GeV}$ staus, hundreds and tens of sleptons may be trapped each year in 10 kton and 1 kton traps, respectively.

These results may be improved significantly if long-lived NLSP sleptons are kinematically accessible at the ILC. For the identical case with $219 \mathrm{GeV}$ sleptons discussed above, $\mathcal{O}(1000)$ sleptons may be trapped each year in a $10 \mathrm{kton}$ trap. If only the NLSP is accessible, this result may be achieved by tuning the beam energy so that produced NLSPs barely escape the ILC detector. The ability to prepare initial states with well-known energies and the flexibility to tune this energy are well-known advantages of the ILC. Here, these features are exploited in a qualitatively new way to produce slow sleptons that are easily captured.

If there are additional superpartner states accessible at the ILC, even tuning the beam energy is not necessary. The cascade decays of other superpartner states produce a broad distribution of slepton energies, and so for a broad range of beam energies, some sleptons will be captured in the trap. We have noted also that, by considering the slightly more general possibility of placing lead or other dense material between the ILC detector and the slepton trap, an order of magnitude enhancement may be possible, allowing up to $\mathcal{O}\left(10^{4}\right)$ sleptons to be trapped per ILC year. 
The analysis here is valid with minor revisions for traps composed of any material. For concreteness, however, we have considered traps composed of water tanks, with the expectation that sleptons caught in water will be easily concentrated and/or moved to quiet environments.

These results imply that high precision studies of slepton decays may be possible. There are many significant implications of such studies. These have been considered in detail in Refs. [6, 10]. Briefly, simply by counting the number of slepton decays as a function of time, the slepton lifetime may be determined with high accuracy. Given thousands of sleptons, we expect a determination at the few percent level. The slepton decay width of Eq. (51) is a simple function of the slepton and gravitino masses, and the slepton mass will be constrained by analysis of the collider event kinematics, a measurement of the slepton width therefore implies a high precision measurement of the gravitino mass and, through Eq. (11), the supersymmetry breaking scale $F$. Such measurements will provide precision determinations of the relic density of superWIMP gravitino dark matter, the contribution of supersymmetry breaking to vacuum energy, and the opportunity for laboratory studies of late decay phenomena relevant for Big Bang nucleosynthesis and the cosmic microwave background.

The gravitino mass may also be determined, although not necessarily on an event-byevent basis, by measuring the energy of slepton decay products. This provides a consistency check. Alternatively, these two methods, when combined, determine not only $m_{\tilde{G}}$, but also the Planck mass $M_{*}$. This then provides a precision measurement of Newton's constant on unprecedentedly small scales, and the opportunity for a quantitative test of supergravity relations.

\section{Acknowledgments}

We are grateful to H. Murayama for collaboration during the early stages of this work and also to H.-C. Cheng, S. Su and F. Takayama for valuable conversations. We thank A. Lankford, W. Molzon, F. Moortgat, D. Stoker, and especially D. Casper for experimental insights. The work of JLF was supported in part by National Science Foundation CAREER Grant PHY-0239817, and in part by the Alfred P. Sloan Foundation.

Note added: E-print hep-ph/0409248 39], in which long-lived sleptons at colliders are also studied, appeared as this work was being finalized. This paper studies the possibility of using an active detector and also considers the idea of using $e^{-} e^{-}$collisions at the ILC. The possibility of transporting trapped sleptons to a low background environment, and the material-independent analysis of optimizing trap shape and placement, both discussed at length here, were not addressed.

[1] For a review, see J. L. Feng, eConf C0307282, L11 (2003) hep-ph/0405215.

[2] J. L. Feng, A. Rajaraman and F. Takayama, Phys. Rev. Lett. 91, 011302 (2003) hep-ph/0302215.

[3] J. L. Feng, A. Rajaraman and F. Takayama, Phys. Rev. D 68, 063504 (2003) hep-ph/0306024.

[4] J. L. Feng, A. Rajaraman and F. Takayama, Phys. Rev. D 68, 085018 (2003) hep-ph/0307375. 
[5] J. R. Ellis, K. A. Olive, Y. Santoso and V. C. Spanos, Phys. Lett. B 588, 7 (2004) hep-ph/0312262.

[6] W. Buchmuller, K. Hamaguchi, M. Ratz and T. Yanagida, Phys. Lett. B 588, 90 (2004) hep-ph/0402179.

[7] J. L. Feng, S. Su and F. Takayama, Phys. Rev. D 70, 063514 (2004) hep-ph/0404198.

[8] J. L. Feng, S. Su and F. Takayama, Phys. Rev. D 70, 075019 (2004) hep-ph/0404231.

[9] F. Wang and J. M. Yang, Eur. Phys. J. C 38, 129 (2004) hep-ph/0405186.

[10] J. L. Feng, A. Rajaraman and F. Takayama, Gen. Rel. Grav. 36, 2575 (2004) hep-th/0405248.

[11] J. R. Ellis, K. A. Olive, Y. Santoso and V. C. Spanos, hep-ph/0408118.

[12] L. Roszkowski and R. R. de Austri, hep-ph/0408227.

[13] For other supersymmetric scenario with extremely long-lived particles, see L. Covi, J. E. Kim and L. Roszkowski, Phys. Rev. Lett. 82, 4180 (1999) hep-ph/9905212 ; L. Covi, H. B. Kim, J. E. Kim and L. Roszkowski, JHEP 0105, 033 (2001) hep-ph/0101009; X. J. Bi, M. z. Li and X. m. Zhang, Phys. Rev. D 69, 123521 (2004) hep-ph/0308218.

[14] M. Kawasaki and T. Moroi, Astrophys. J. 452, 506 (1995) astro-ph/9412055.

[15] E. Holtmann, M. Kawasaki, K. Kohri and T. Moroi, Phys. Rev. D 60, 023506 (1999) hep-ph/9805405.

[16] M. Kawasaki, K. Kohri and T. Moroi, Phys. Rev. D 63, 103502 (2001) hep-ph/0012279.

[17] R. H. Cyburt, J. R. Ellis, B. D. Fields and K. A. Olive, Phys. Rev. D 67, 103521 (2003) astro-ph/0211258.

[18] K. Jedamzik, Phys. Rev. D 70, 063524 (2004) astro-ph/0402344.

[19] M. Kawasaki, K. Kohri and T. Moroi, astro-ph/0402490 astro-ph/0408426.

[20] M. Fukugita and T. Yanagida, Phys. Lett. B 174, 45 (1986).

[21] W. Buchmuller, P. Di Bari and M. Plumacher, Nucl. Phys. B 665, 445 (2003) hep-ph/0302092.

[22] T. Moroi, H. Murayama and M. Yamaguchi, Phys. Lett. B 303, 289 (1993).

[23] M. Bolz, A. Brandenburg and W. Buchmuller, Nucl. Phys. B 606, 518 (2001) hep-ph/0012052.

[24] M. Bolz, W. Buchmuller and M. Plumacher, Phys. Lett. B 443, 209 (1998) hep-ph/9809381.

[25] M. Fujii, M. Ibe and T. Yanagida, Phys. Lett. B 579, 6 (2004) hep-ph/0310142.

[26] R. Allahverdi and M. Drees, Phys. Rev. D 70, 123522 (2004) hep-ph/0408289.

[27] A non-supersymmetric, but similar, scenario with long-lived states has been investigated in J. L. Goity, W. J. Kossler and M. Sher, Phys. Rev. D 48, 5437 (1993) hep-ph/9305244.

[28] F. Cerutti, M. Fanti, P. Giacomelli, P. Kluit, R. McNeil, C. Rembser and U. Schwickerath, LEPSUSYWG/02-05.1.

[29] G. Belanger, F. Boudjema, A. Cottrant, A. Pukhov and A. Semenov, hep-ph/0407218

[30] For a review, see J. F. Ziegler, J. Appl. Phys 85, 1249 (1999).

[31] H. Bichsel, D. E. Groom and S. R. Klein, Phys. Rev. D 66, 010001 (2002).

[32] H. Tai, H. Bichsel, J. Wilson, J. Shinn, F. Cucinotta and F. Badavi, NASA Technical Paper 3644 .

[33] B. Schorr, Comp. Phys. Comm. 7, 215 (1974).

[34] Atlas Technical Proposal, http://atlas.web.cern.ch/Atlas/TP/tp.html.

[35] CMS Technical Proposal, http://hep.fuw.edu.pl/cms/tp/tp.html

[36] F. E. Paige, S. D. Protopescu, H. Baer and X. Tata, hep-ph/0312045.

[37] R. Brinkmann, K. Flottmann, J. Rossbach, P. Schmuser, N. Walker and H. Weise, "TESLA: The superconducting electron positron linear collider with an integrated X- 
ray laser laboratory. Technical design report. Pt. 2: The accelerator," DESY-01-011, http://www.desy.de/ lcnotes/tdr.

[38] T. Abe et al. [American Linear Collider Working Group Collaboration], "Linear collider physics resource book for Snowmass 2001. 1: Introduction," in Proc. of the APS/DPF/DPB Summer Study on the Future of Particle Physics (Snowmass 2001) ed. N. Graf, hep-ex/0106055.

[39] K. Hamaguchi, Y. Kuno, T. Nakaya and M. M. Nojiri, hep-ph/0409248. 